\title{
Backstepping experimentally applied to an antagonistically driven finger with flexible tendons
}

\author{
Maxime Chalon ${ }^{1}$ and Brigitte d'Andréa-Novel ${ }^{2}$ \\ ${ }^{1}$ DLR - Institute of Robotics and Mechatronics, German Aerospace Center (DLR), Germany \\ ${ }^{2}$ Mines ParisTech, CAOR, Paris, France
}

\begin{abstract}
The Hand Arm System of DLR is a complex mechatronic system built to approach the human in terms of dynamics and dexterity. Its fingers are antagonistically actuated by flexible tendons, resulting in a nonlinear flexible joint. The advantages of such a design are the high dynamics but more importantly the enhanced robustness. Nonlinear control methods have been developed in the community that are, at least in principle, applicable to such systems. However, because of the complexity of some methods, most works are focusing on simulations and do not consider the practical issues arising with hardware. Such issues are, for example, the need for derivatives or the use of large matrix inversions. In this paper, we adapt the backstepping method to the specific case of an antagonistic actuation. The modeling of the mechanism is followed by the design of the controller and the work is concluded by a set of experimental results on the hand of the Hand Arm System, the "Awiwi hand".
\end{abstract}

\section{INTRODUCTION}

Tendon driven mechanisms are a common choice in the design of robotic hands. It is anthropomorphic, but more importantly, the remote actuation provides a low link inertia, a small form factor for the link side and high dynamics. Moreover, in conjunction with variable stiffness elements in an antagonistic configuration, it is possible to control position and stiffness of the joints independently. In [1], the advantages and drawbacks of variable stiffness are discussed. In [2], AlbuSchäffer et al. highlight that the intrinsic compliance seen as a drawback in the past, can be a feature today. For small robots, such as hands, it is especially interesting in order to protect the robot itself. Indeed, since a control law cannot react in the very first instant of the impact, the only protection of the robot is its intrinsic compliance.

This paper focuses on the control of a joint driven by antagonistic tendon with nonlinear stiffness.

The control of flexible joints was treated in more depth. Previous works, such as [3], [4], consider ranges of stiffness and inertia that are not comparable with the ones found in fingers. Their assumptions or simplifications are therefore not always applicable to the Awiwi hand. Adaptive motor level PD controller was presented in [5]. Passitivity based impedance controllers for the case of constant stiffness was treated in [6]. The most intuitive approach is probably a singular perturbation approach [7]. It relies on an assumption on the time scale difference between the link side and the

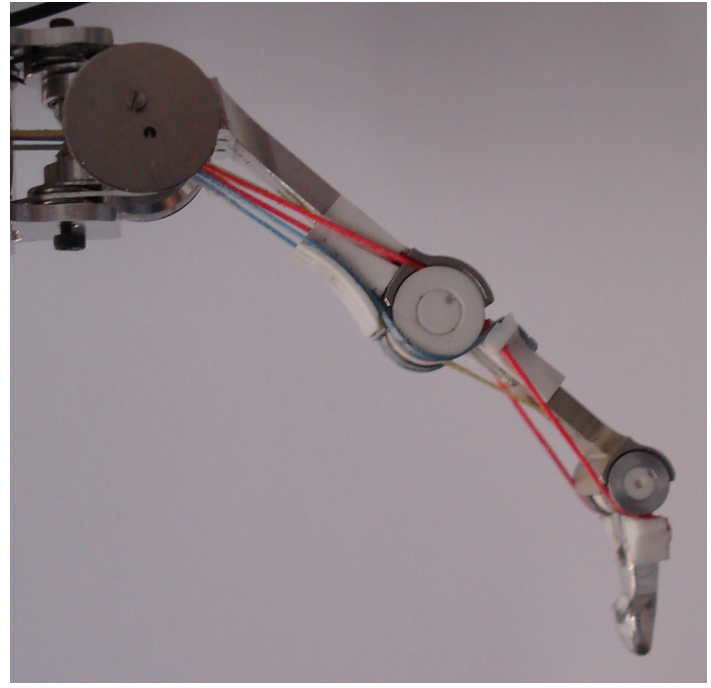

Fig. 1. The index finger of the Awiwi hand, the hand of the DLR Integrated Hand Arm System (4 DOF, 8 tendons)

motor side; but may not hold if the inertia or stiffness is varying over a large range. A cascaded approach [8] is also very natural and does not require the time scale assumptions, however it is not a constructive method so the stability proof must be devised in a separate step. Finally, nonlinear methods have been proposed in the literature such as backstepping [9], feedback linearization [10], [11] or sliding mode control [12], [13]. Dynamic surface control [14] is an extension of the backstepping that accounts explicitly for the effects of filters that are used to obtain high derivatives. It requires solving a nonlinear matrix inequality problem of twice the dimension of the system.

Works on tendon driven system focused on linear elasticity in the context on serial elastic actuators (SEA). A neural network based controller for flexible joint was proposed in [15], [16]. More recently the implementation and control of the Robonaut tendon driven fingers were presented in [17], [18]. A controller for a variable stiffness mechanism using two motors and two springs in an antagonistic configuration is presented in [19] but the control is limited to a simple PD approach. In [20], the impedance control scheme for variable stiffness 
mechanisms with nonlinear joint with a specific model was presented. ${ }^{1}$. Feedback linearization of uncoupled joints that are each driven antagonistically was treated in [21], [22]. Most nonlinear method require high order derivatives for the tracking case. They are rarely directly measured therefore the need for filtering is critical.

This work considers the regulation case since it is the most relevant application case for a robotic hand. It proposes a controller that maintains the symmetry of the problem. The main contribution of this paper is to derive, apply and verify an integrator backstepping controller for the finger of the Awiwi hand. The experimental results, demonstrate the performance of the method with respect to a linear approach.

The modeling of the tendon driven finger is proposed in the first section. The second section details the design of the backtepping controller to the specific case of an antagonistic actuation. First a link side impedance controller is designed. Then, a decoupling step is applied to allow for a Single Input Single Output (SISO) design of the motor controllers. Finally, the backstepping method is applied to each motor. Last section describes several experimental setups and reports the results. It is shown that the method presents a clear advantage over previously applied methods. In particular, the method does not require assumption regarding the time scales, as it is the case for a singular perturbation approach.

\section{Modeling}

In this section, the dynamic equations for the index finger, which is driven by a set of antagonistic and nonlinear elastic tendon, are derived using CAD data. The model is referenced in the literature as the flexible joint model [23]-[25]. This paper focuses on the index finger but the approach applies similarly to the other fingers since they have a similar mechanical structure. In the Awiwi hand, the thumb uses a tensegrity structure together with nonlinear elastic tendons. Its modeling has been reported in [26].

The index finger Fig. 2 has 4 degrees of freedom (DOF) that are driven by 8 tendons. The base joint is driven by four tendons. The Proximal Interphalangeal (PIP) and Distal Interphalangeal(DIP) joints are driven by four more tendons. The latter tendons are nearly going through the rotation center of the Metacarpal Proximal (MCP), and therefore have a negligible influence on the base torques.

The coupling between the dynamics of the motors and the link side is limited to the tendon forces. Therefore, the dynamic equations are obtained by coupling the dynamics of the eight Permanent Magnet Synchronous Motors (PMSM), with those of a serial robot with four joints. The diagram of Fig. 3 depicts the structure of the mechanism. Similar to the approach used in [20], the equations are:

$$
\begin{cases}\boldsymbol{M}(\boldsymbol{q}) \ddot{\boldsymbol{q}}+\boldsymbol{C}(\dot{\boldsymbol{q}}, \boldsymbol{q}) \dot{\boldsymbol{q}}+\boldsymbol{g}(\boldsymbol{q})+\boldsymbol{\tau}_{\mathrm{fric}, \mathrm{q}} & =\boldsymbol{P}(\boldsymbol{q}) \boldsymbol{f}_{\mathrm{t}}(\boldsymbol{q}, \boldsymbol{\theta})+\boldsymbol{\tau}_{\mathrm{ext}} \\ \boldsymbol{B} \ddot{\boldsymbol{\theta}}+\boldsymbol{E} \boldsymbol{f}_{\mathrm{t}}(\boldsymbol{q}, \boldsymbol{\theta}) & =\boldsymbol{\tau}_{\mathrm{m}}\end{cases}
$$

where the variables are defined according to Table I. Following the conventions of [27], [28], the coupling matrix $\boldsymbol{P}(\boldsymbol{q}) \in$

\footnotetext{
${ }^{1}$ The controller requires a linear change of stiffness w.r.t the tendon force that is $k=\alpha f$
}

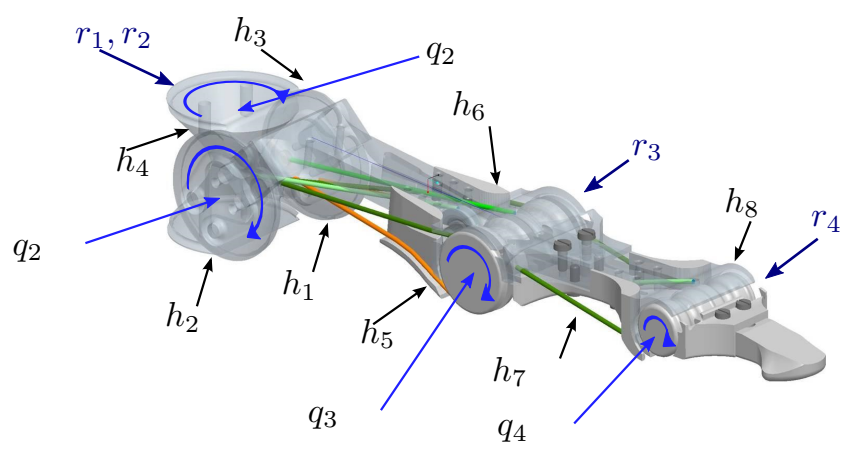

Fig. 2. Index model: four joints and their associated 8 tendons

TABLE I

DESCRIPTION OF VARIABLES

\begin{tabular}{|c||l|}
\hline Symbol & Designation \\
$m \in \in \mathbb{N}$ & number of joints \\
$\boldsymbol{h} \in \mathbb{R}^{m}$ & number of tendons \\
$\boldsymbol{q} \in \mathbb{R}^{n}$ & positions of the tendon w.r.t. a fixed reference \\
$\boldsymbol{\tau}_{\mathrm{q}} \in \mathbb{R}^{n}$ & positions of the joint \\
$\boldsymbol{f}_{\mathrm{t}} \in \mathbb{R}^{m}$ & joint torques generated by the tendon forces \\
$\boldsymbol{K}_{\mathrm{t}} \in \mathbb{R}^{m \times m}$ & forces applied on the tendon \\
$\boldsymbol{K}_{\mathrm{q}} \in \mathbb{R}^{n \times n}$ & stiffness matrix of the tendons \\
$k_{\mathrm{t}_{\mathrm{i}}}\left(\boldsymbol{f}_{\mathrm{t}}\right) \in \mathbb{R}$ & stiffness matrix of the joints \\
$\boldsymbol{P}(\boldsymbol{q}) \in \mathbb{R}^{n \times m}$ & stiffness of the tendon depending on the tendon \\
$\boldsymbol{E} \in \mathbb{R}^{m \times m}$ & force \\
$\boldsymbol{M}(\boldsymbol{q}) \in \mathbb{R}^{n \times n}$ & coupling matrix \\
$\boldsymbol{B} \in \mathbb{R}^{m \times m}$ & motor pulley radii matrix \\
$\boldsymbol{C}(\dot{q}, q)$ & link side mass matrix \\
$\boldsymbol{g}(q) \in \mathbb{R}^{n}$ & diagonal motor side mass matrix \\
$\boldsymbol{\tau}_{\text {fric }, \mathrm{q}} \in \mathbb{R}^{n}$ & link side gravity vector \\
$\boldsymbol{\tau}_{\mathrm{m}} \in \mathbb{R}^{m}$ & frictional link torque \\
\hline \multicolumn{2}{|c}{} \\
\hline
\end{tabular}

$\mathbb{R}^{n \times m}$, relates the $m$ tendon velocities $\dot{h} \in \mathbb{R}^{m}$ to the $n$ joint velocities $\dot{\boldsymbol{q}} \in \mathbb{R}^{n}$. It is obtained as the derivative of the tendon position $\boldsymbol{h}$ with respect to the joint position $\boldsymbol{q}$ that is,

$$
\boldsymbol{P}^{T}(\boldsymbol{q})=\frac{\partial \boldsymbol{h}(\boldsymbol{q})}{\partial \boldsymbol{q}} .
$$

Using the fact the work of the tendons is equal to the work of the joint (the principle of virtual work), one obtains

$$
\boldsymbol{\tau}_{\mathrm{q}}=\boldsymbol{P}(\boldsymbol{q}) \boldsymbol{f}_{\mathrm{t}}(\boldsymbol{q}, \boldsymbol{\theta}) .
$$

The tendon elongation depends on the tendon force. The relationship is generated by the Flexible Antagonistic Spring (FAS) mechanism depicted in Fig. 4, see [29]. The motivation to use such a mechanism has been reported in [30]. Due to the mechanism, the stiffness of a tendon depends on the tendon force. It is locally obtained as:

$$
k_{\mathrm{t}}\left(\boldsymbol{q}_{0}, \boldsymbol{\theta}_{0}\right)=\left.\frac{\partial \boldsymbol{f}_{\mathrm{t}}(\boldsymbol{q}, \boldsymbol{\theta})}{\partial \boldsymbol{h}(\boldsymbol{q}, \boldsymbol{\theta})}\right|_{q=q_{0}, \theta=\theta_{0}} .
$$

Since, in the index finger of the Awiwi hand the tendon are routed independantly, the tendon stiffness can be grouped to form a diagonal matrix.

$$
\boldsymbol{K}_{\mathrm{t}} \in \mathbb{R}^{m \times m} .
$$

It is crucial to note that the equations hold only as long as the tendon forces are in their prescribed working range $f \in$ 


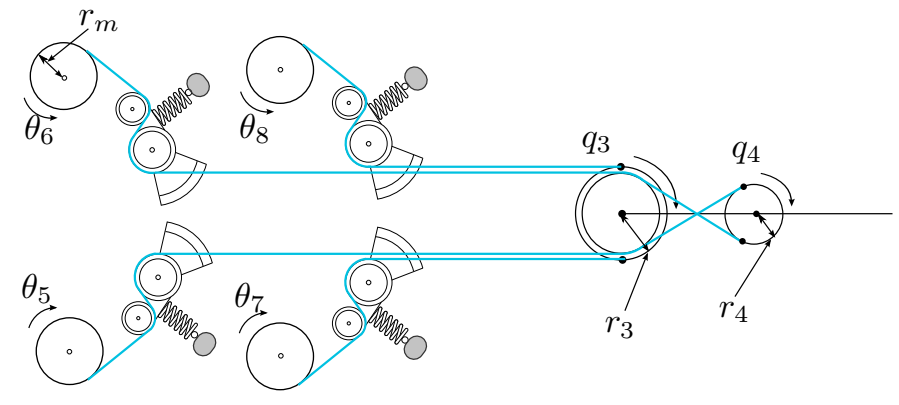

Fig. 3. Diagram: PIP and DIP joints of the index, two joints and their associated four tendons

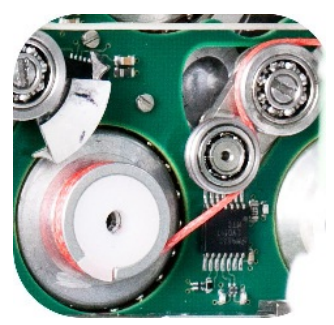

Fig. 4. Picture of the FAS mechanism

$\left[f_{\min } . . f_{\max }\right]$. In the rest of the paper this assumption is considered to be satisfied.

\section{Controller Design}

The availability of an impedance controller at joint level is nearly a prerequisite for research on grasping. It allows to conform to the object and enables grasp teaching in a very intuitive way. It is paramount to control the tendon force to avoid tendon damage or slack. The behavior of the motors is of little interest except maybe for the noise or efficiency. The first step eliminates the inertial coupling between the two motors and the link by a partial feedback linearization. The second step presents the design of the impedance controller at joint level. That is, the link behavior in terms of stiffness and damping is specified. Third, the tendon controller is designed by using the backstepping method. The estimation of the link position and torque, required for the impedance controller, was presented in [26] and is not repeated herein. The equation are reported for a single joint so as to ease the notations and are applicable to a multi-DOF system in the regulation case.

\section{A. Inertia Decoupling}

The considered system is a multiple input single output (MISO) system. That is, two motors are used to actuate a single joint. However, the backstepping method is best applied to a SISO system although extensions to MISO system have been proposed [31]. Different approaches are available to transform the system, for example the scheme of pusherfollower [11] or a decoupling approach. In the present case, in order to keep the symmetry of the system, it has been decided to treat each motor independently. A partial feedback linearization cancels the inertial coupling between the motor and the nonlinear stiffness terms. Applied to a single joint (1) is more clearly written as:

$$
\begin{aligned}
& b_{\mathrm{m}} \ddot{q}=\tau_{1}-\tau_{2}+\tau_{\mathrm{ext}} \\
& b_{1} \ddot{\theta}_{1}=-\tau_{1}+\tau_{r_{\mathrm{q}} \mathrm{m}, 1}, \\
& b_{2} \ddot{\theta}_{2}=-\tau_{2}+\tau_{r_{\mathrm{q}} \mathrm{m}, 2}
\end{aligned}
$$

where $\left(\theta_{1}, \theta_{2}\right) \in \mathbb{R}^{2}, q \in \mathbb{R}$ are the motor positions and link position. The link inertia and the motor inertia are denoted $b_{\mathrm{m}} \in \mathbb{R}, b_{1} \in \mathbb{R}$ and $b_{2} \in \mathbb{R}$. The radius of the motor pulley and the joint pulley are denoted $\left(r_{\mathrm{m}}, r_{\mathrm{q}}\right) \in \mathbb{R}^{2}$. The joint torque generated by the tendons are $\tau_{1}=k_{1}\left(r_{\mathrm{m}} \theta_{1}-\right.$ $\left.r_{\mathrm{q}} q\right)\left(r_{\mathrm{m}} \theta_{1}-r_{\mathrm{q}} q\right)$ and $\tau_{2}=k_{2}\left(r_{\mathrm{m}} \theta_{2}+r_{\mathrm{q}} q\right)\left(r_{\mathrm{m}} \theta_{2}+r_{\mathrm{q}} q\right)$. In the following, it is assumed that $b_{1}=b_{2}=b, r_{\mathrm{m}}=r_{\mathrm{q}}=1$ and $k_{1}=k_{2}=k$ in order to simplify the notations. Defining $\phi_{1}$ as the elongation of the first tendon, that is $\phi_{1}=\left(r_{\mathrm{m}} \theta_{1}-r_{\mathrm{q}} q\right)$ and deriving it twice, with respect to time, yields

$$
\begin{array}{lcc}
\tau_{1}= & k\left(\phi_{1}\right) \phi_{1} \\
\dot{\tau}_{1}= & \frac{\partial k\left(\phi_{1}\right)}{\partial \phi_{1}} \dot{\phi}_{1}+k\left(\phi_{1}\right) \dot{\phi_{1}} \\
\ddot{\tau}_{1}= & \frac{\partial^{2} k\left(\phi_{1}\right)}{\partial \phi_{1}^{2}} \dot{\phi}_{1}{ }^{2}+\frac{\partial k\left(\phi_{1}\right)}{\partial \phi_{1}} \ddot{\phi}_{1}+\frac{\partial k\left(\phi_{1}\right)}{\partial \phi_{1}} \dot{\phi}_{1}{ }^{2}+k\left(\phi_{1}\right) \ddot{\phi}_{1} \\
\ddot{\tau}_{1}= & \Gamma\left(\phi_{1}, \dot{\phi}_{1}\right)+k\left(\phi_{1}\right)\left(\ddot{\theta}_{1}-\ddot{q}\right) \\
\ddot{\tau}_{1}= & \Gamma+\frac{k\left(\phi_{1}\right)}{b}\left(-\tau_{1}+\tau_{\mathrm{m}, 1}\right)-\frac{k\left(\phi_{1}\right)}{b_{\mathrm{m}}}\left(\tau_{1}-\tau_{2}+\tau_{\text {ext }}\right)
\end{array}
$$

where $\Gamma(\phi, \dot{\phi})$ contains all the nonlinear terms. It follows that (7) can be decoupled with a feedback linearization

$$
\tau_{\mathrm{m}, 1}=\tau_{1}+\frac{b}{k}\left(-\Gamma\left(\phi_{1}, \dot{\phi}_{1}\right)-\frac{k}{b_{\mathrm{m}}}\left(\tau_{1}-\tau_{2}\right)\right) .
$$

The expression of $\tau_{\mathrm{m}, 2}$ is obtained by symmetry and applying the feedback yields

$$
\begin{aligned}
& \ddot{\tau}_{1}=u_{1} \\
& \ddot{\tau}_{2}=u_{2}
\end{aligned}
$$

One can remark that it is only required to perform a linearization of the coupling terms since the backstepping procedure incorporates the rest of the linearization. In this paper, the complete linearization of (7) is performed to simplify the backstepping integration equations of the following sections. Although the tendon are unable to push, releasing the tendon is equivalent to pushing a tendon. That is, as long as the internal pretension is positive, each of the tendons can generate positive and negative joint torque. In the nominal case, all tendon forces are well within the workspace and all requirements are satisfied. The dynamic equation are partially invalid and the controller is not guaranteed to be stable anymore if the tendon forces reach the workspace limits. In such a case, a proper handling scheme is necessary but is not reported here.

\section{B. Torque distribution}

The joint torque generated by the action of both tendons should satisfy

$$
\tau_{\mathrm{q}, \mathrm{des}}=\tau_{1, \mathrm{des}}-\tau_{2, \mathrm{des}}=-K_{\mathrm{p}, \mathrm{imp}} q-K_{\mathrm{d}, \mathrm{imp}} \dot{q},
$$


since a regulation impedance behavior of the link side is desired. The torques generated by the tendons are subject to the constraint

$$
\left(\tau_{1}, \tau_{2}\right) \in S^{2}
$$

where $S \subset \mathbb{R}$ is a real compact subspace given by the maximum and minimum tendon force. According to (10) there exists many combinations of motor torques that generate the desired joint torque. The choice

$$
\begin{aligned}
& \tau_{1, \text { des }}=\tau_{1, \text { offset }}+0.5 \tau_{\mathrm{q}, \text { des }} \\
& \tau_{2, \text { des }}=\tau_{2, \text { offset }}-0.5 \tau_{\mathrm{q}, \text { des }}
\end{aligned}
$$

where $\tau_{1, \text { des }}$ and $\tau_{2 \text {,des }}$ denote the desired link torque to be produced by the action of each tendon, is a choice that symmetrically shares the torque. It is important to note that the internal pretension is a degree of freedom that can be used to satisfy auxiliary goals such as a mechanical link stiffness. Bio-inspired methods for the stiffness adjustement have been discussed for a finger in [32]. It is mostly depending on the application and is not adjusted online, thus it has no influence on the stability. The analysis presented herein assumes that the reference stiffness is varied slowly or is fixed, that is, the derivative of $\tau_{1 \text {,offset }}$ does not appear in the Lyapunov analysis.

\section{Strict feedback form}

Thanks to the symmetry of the problem, one can concentrate on a single motor, thus the following section treats the case of $\theta_{1}$. The backstepping is a recursive procedure that propagates the control error downward by successively building Lyapunov functions and selecting intermediate control laws. The procedure ends when the system input is reached therefore the system must be triangular to ensure that the procedure ends. Defining the state vector $\boldsymbol{x} \in \mathbb{R}^{4}$ as $\boldsymbol{x}=\left[x_{1}=q, x_{2}=\right.$ $\left.\dot{q}, x_{3}=\tau_{1}, x_{4}=\dot{\tau}_{1}\right]^{T}$, results in a strict feedback form description

$$
\begin{array}{cc}
\ddot{x}_{1}= & b_{\mathrm{m}}^{-1}\left(x_{3}+\tau_{\text {ext }}\right) \\
\dot{x}_{3}= & x_{4} \\
\dot{x}_{4}= & u_{1}
\end{array}
$$

\section{Reference control law}

The two motors have been decoupled so the problem is treated as a set of independent differential systems. Let $V_{2}\left(x_{1}, x_{2}\right)$, be the Lyapunov function

$$
V_{2}\left(x_{1}, x_{2}\right)=\frac{1}{2} b_{\mathrm{m}} x_{2}^{2}+\frac{1}{2} K_{\mathrm{p}, \mathrm{imp}} x_{1}^{2}
$$

where $K_{\mathrm{p}, \mathrm{imp}} \in \mathbb{R}$ is a positive scalar. The time derivative along the solution of (13) is

$$
\dot{V}_{2}\left(x_{1}, x_{2}\right)=x_{2}\left(x_{3}+\tau_{\text {ext }}\right)+K_{\mathrm{p}, \mathrm{imp}} x_{1} x_{2} .
$$

The torque reference for $x_{3}$ is denoted $\bar{x}_{3}$ and is selected according to (12) in order to yield an impedance behavior of the link side

$$
\bar{x}_{3}=-K_{\mathrm{p}, \mathrm{imp}} x_{1}-K_{\mathrm{d}, \mathrm{imp}} x_{2},
$$

where $K_{\mathrm{p}, \mathrm{imp}}>0$ (resp. $K_{\mathrm{d}, \mathrm{imp}}>0$ ) is the impedance controller stiffness (resp. the impedance controller damping).
A first error must be introduced to account for the fact that the tendon forces cannot be adjusted instantaneously. The first tracking error $z_{3} \in \mathbb{R}$, which is a joint torque error, is defined as

$$
z_{3}=x_{3}-\bar{x}_{3}
$$

1) First backstep: Writing the system (13) in terms of the error $z_{3}$ yields

$$
\begin{array}{cc}
b_{\mathrm{m}} \ddot{x}_{1}= & -K_{\mathrm{p}, \mathrm{imp}} x_{1}-K_{\mathrm{d}, \mathrm{imp}} x_{2}+\tau_{\mathrm{ext}}+z_{3} \\
\dot{z}_{3}= & x_{4}-\dot{\bar{x}}_{3} \\
\dot{x}_{4}= & u_{1}
\end{array}
$$

Defining the Lyapunov function

$$
V_{3}\left(x_{1}, x_{2} z_{3}\right)=\frac{1}{2}\left(b_{\mathrm{m}} x_{2}^{2}+K_{\mathrm{p}, \mathrm{imp}} x_{1}^{2}+z_{3}^{2}\right),
$$

it follows

$$
\dot{V}_{3}\left(x_{2}, x_{4} z_{3}\right)=-K_{\mathrm{d}, \mathrm{imp}} x_{2}^{2}+z_{3}\left(x_{4}-\dot{\bar{x}}_{3}\right) .
$$

Selecting the reference $\bar{x}_{4}=-x_{2}+\dot{\bar{x}}_{3}-k_{3} z_{3}$, where $k_{3}>0$ is a gain used to accelerate the torque convergence, for $x_{4}$ and introducing the error $z_{4}=x_{4}-\bar{x}_{4}$, the Lyapunov derivative becomes

$$
\dot{V}_{3}\left(x_{2}, x_{4} z_{3}\right)=-K_{\mathrm{d}, \mathrm{imp}} x_{2}^{2}-k_{3} z_{3}^{2}+z_{3} z_{4},
$$

which is negative semi-definite apart for the last term.

2) Second backstep: System (18) in terms of the error $z_{4}$ is given by

$$
\begin{array}{cc}
b_{\mathrm{m}} \ddot{x}_{1}= & -K_{\mathrm{p}, \mathrm{imp}} x_{1}-K_{\mathrm{d}, \mathrm{imp}} x_{2}+\tau_{\mathrm{ext}}+z_{3} \\
\dot{z}_{3}= & -x_{2}-k_{3} z_{3}+z_{4} \\
\dot{z}_{4}= & u_{1}-\dot{\bar{x}}_{4}
\end{array}
$$

Let $V_{4}\left(x_{1}, x_{2} z_{3}, z_{4}\right)$ be the Lyapunov function defined by

$$
V_{4}\left(x_{1}, x_{2} z_{3}, z_{4}\right)=\frac{1}{2}\left(b_{\mathrm{m}} x_{2}^{2}+K_{\mathrm{p}, \mathrm{imp}} x_{1}^{2}+z_{3}^{2}+z_{4}^{2}\right) .
$$

Its derivative along the solution of (22) is

$$
\dot{V}_{4}\left(x_{2}, z_{3}, z_{4}\right)=-K_{\mathrm{d}, \mathrm{imp}} x_{2}^{2}-k_{3} z_{3}^{2}+z_{3} z_{4}+z_{4}\left(u_{1}-\dot{\bar{x}}_{4}\right) .
$$

Selecting $u_{1}=-z_{3}+\dot{\bar{x}}_{4}-k_{4} z_{4}$, where $k_{4}>0$ is a gain to accelerate the convergence, the Lyapunov derivative is simplified in

$$
\dot{V}_{4}\left(x_{2}, z_{3} z_{4}\right)=-K_{\mathrm{d}, \mathrm{imp}} x_{2}^{2}-k_{3} z_{3}^{2}-k_{4} z_{4}^{2},
$$

which is negative semi-definite and achieves the controller construction.

3) Input expression: The input expression is obtained by recursively replacing the expression of the errors and the references. It is important to note that some terms, such as $\dot{\bar{x}}_{3}$ or $\dot{\bar{x}}_{4}$ contain high order derivatives. It is better to use the analytical expression of those derivatives rather than a discrete implementation in order to avoid amplifying the noise. Similarly, during simulation, the use of integrators and derivatives should be carefully analyzed to reach a satisfying simulation speed. 


\section{E. Discussion}

In this section, the backstepping method has been applied to the case of an antagonistic actuation. A sharing of the desired joint torque allowed to derive two symmetric controllers. The asymptotic stability is guaranteed by the choice of the gains as long as the tendon forces remain positive. Thanks to the symmetry, the stiffness of the joint is naturally introduced as a shifting of the desired working point for the tendon preload. It is worth noting that in case of failure of one of the motor, the controller will be able to operate but the controller impedance will not be the prescribed one.

\section{EXPERIMENTAL RESULTS AND SIMULATIONS}

This section reports the experimental results obtained with the real-time system. The implementation is done using MATLAB/Simulink on a QNX operating system. ${ }^{2}$ First, a simulation is performed to verify that the controller behaves as an impedance controller. Second, the controller is applied to a single flexible joint driven by a single motor and using linear springs. Unlike the fingers, the setup allows to use a very low joint stiffness and joint damping to magnify the effets of the backstepping approach. Moreover, it allows the link inertia to be modified and provides a high resolution measure of the link side position. Finally, a joint of the index of the Awiwi hand is used to verify that an impedance behavior is realized and that the control is performing adequately across a large frequency range.

\section{A. Controller behavior}

Before implementing the controller on the real system, a simulation verifies that the controller behaves as a joint impedance controller. Fig. 5 reports the simulation results. The desired link position, the measured link position and the externally applied load are represented. In the first half of the time (i.e. $t<1$ s), a position step is commanded. The link position is critically damped and has a negligible steadystate error. In the second half of the time (i.e. $t>1 \mathrm{~s}$ ), the desired position is set to the origin and two external force steps are successively applied. The disturbance results in a link deflection that is proportional to the applied force, thus confirming that the link behaves like a virtual spring.

\section{B. SISO system with linear stiffness}

A first experiment is proposed on a single motor driving a single joint placed in an horizontal configuration (cf. Fig. 6). Both tendons are attached to the same motor, thus resulting in a SISO system. The tendons are using very soft spring in series in order to highlight the backstepping action. As depicted in Fig. 7, the backstepping controller is able to regulate the position of a flexible joint with low damping. The wave highlighted by $\mathrm{A}$ in Fig. 7, is due to the fact that the joint torque is minimal around the set point. The motor must realize a large displacement to generate a sufficient torque to slow down the link.

\footnotetext{
${ }^{2}$ More details on the hardware communication infrastructure, using BiSS and Spacewire is found in [33].
}

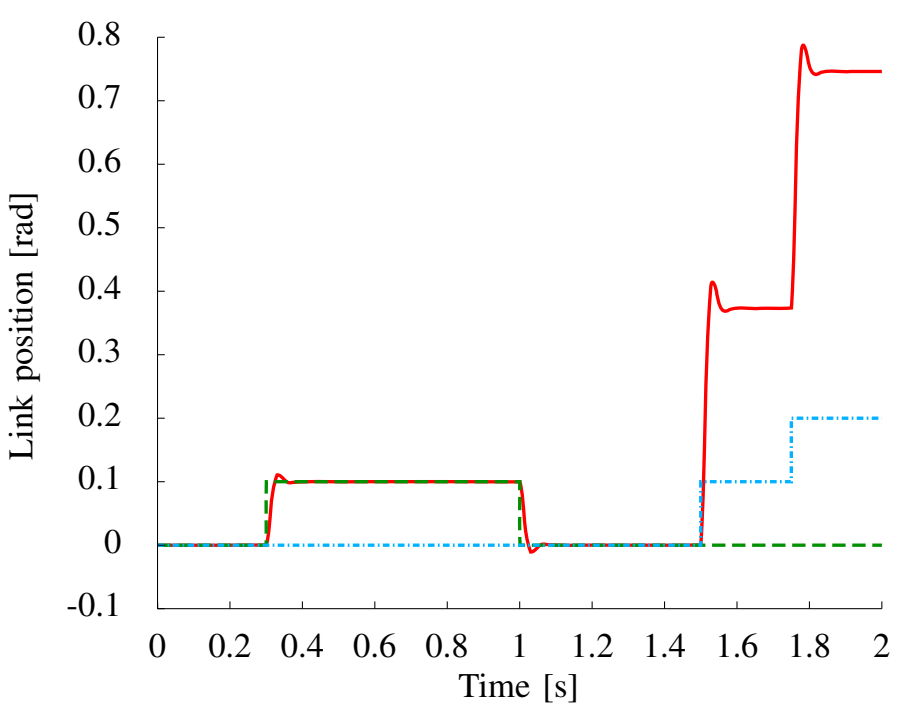

Fig. 5. Simulation antagonistic backstepping. The green dashed line depicts the desired link position. The solid red line represents the link position. The dotted light blue curve represents an externally applied joint torque.

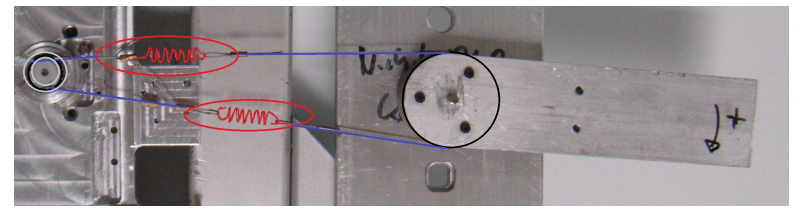

Fig. 6. Photograph of the single motor with single link setup. On the left is the motor, in the middle are two tendons with two linear springs and on the right is the link.

\section{Step response with the finger}

The single motor experiment demonstrates that the controller works in principle. Therefore, the controller equations for an antagonistic joint are applied to the PIP joint of the index (cf. Fig. 1). The results are reported in Fig. 8. The experiment is realized with a $20 \mathrm{~N}$ pretension. It can be seen that the controller successfully moves the link to the desired position. The link is moved in about $20 \mathrm{~ms}$ to the target position with a very slight overshoot. This performance was not possible with a linear controller.

\section{Linearity of the impedance}

The previous experiments show that the controller successfully moves the joint. However, it does not indicate if the impedance stiffness is effectively realized. Therefore, an experiment is performed to evaluate the relationship between the joint torque and the impedance stiffness during an imposed link deflection. First, a joint of the index finger is mechanically attached to a rigid fixture and a known joint deflection is imposed. Then, the stiffness of the impedance controller of the joint is varied and the resulting torque is recorded. The relationship, which would ideally be a straight line, is depicted in Fig. 9. The results confirm that the controller is generating a torque nearly proportional to the controller stiffness. 


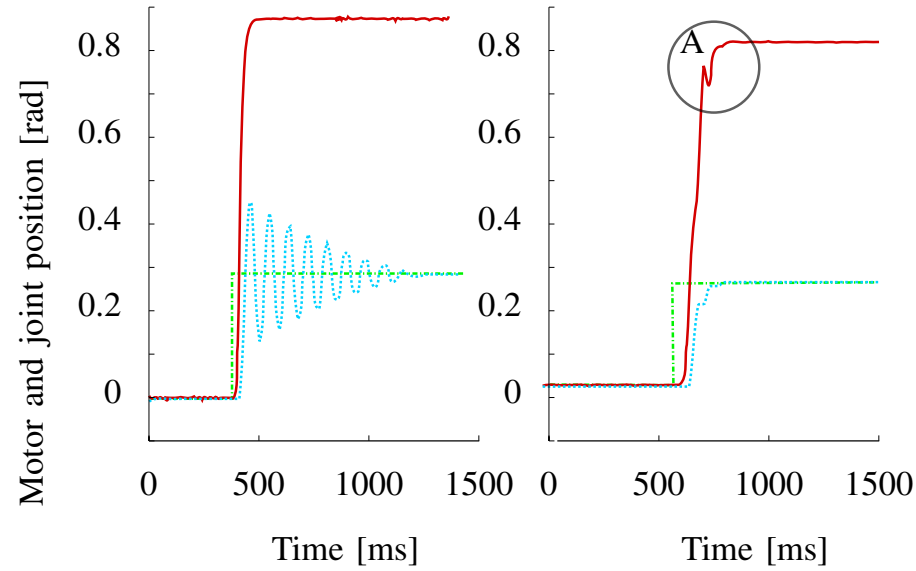

Fig. 7. Measured link position during a step response. The green dashed line depicts the desired link position. The solid red curve depicts the motor position while the dotted light blue curve represents the link position. Left: a motor PD controller is used. Right: the backstepping controller is used. The large link oscillation obtained on the left highlights the link stiffness and the low dampling of the link.

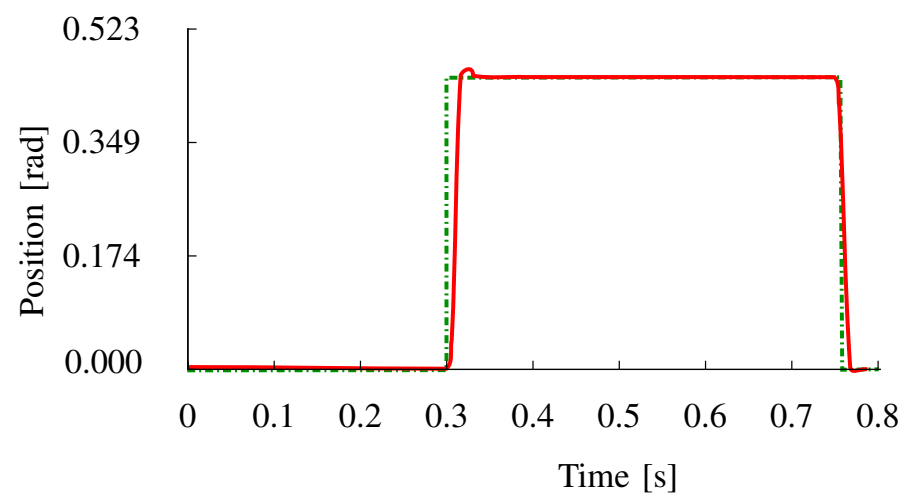

Fig. 8. Measured link position during a step response. The green dashed line depicts the desired link position. The solid red line represents the link position with the backstepping controller.

\section{E. Frequency response comparison}

Finally, the performance of the controller is analysed in term of bandwidth. The gain diagram is obtained by recording the amplification gain of a small sinusoidal position command over a frequency domain. As the system is nonlinear, the frequency response is changing with the pretension, herein the nominal case is reported. The gain diagram reported in Fig. 10 shows that the backstepping controller achieves a unit gain up to $80 \mathrm{rad} / \mathrm{s}$.

\section{CONCLUSION AND FUTURE WORK}

A nonlinear control method, known as the integrator backstepping method was adapted to an antagonistically tendon driven joint, with nonlinear stiffness. In the first section, the modeling of the joint has been presented and the dynamics have been derived. The second section started by decoupling the system in order to apply the backstepping integrator procedure while maintaining the symmetry of the problem. The method heavily relies on partial feedback linearization even in the regulation case. Therefore, although the stability

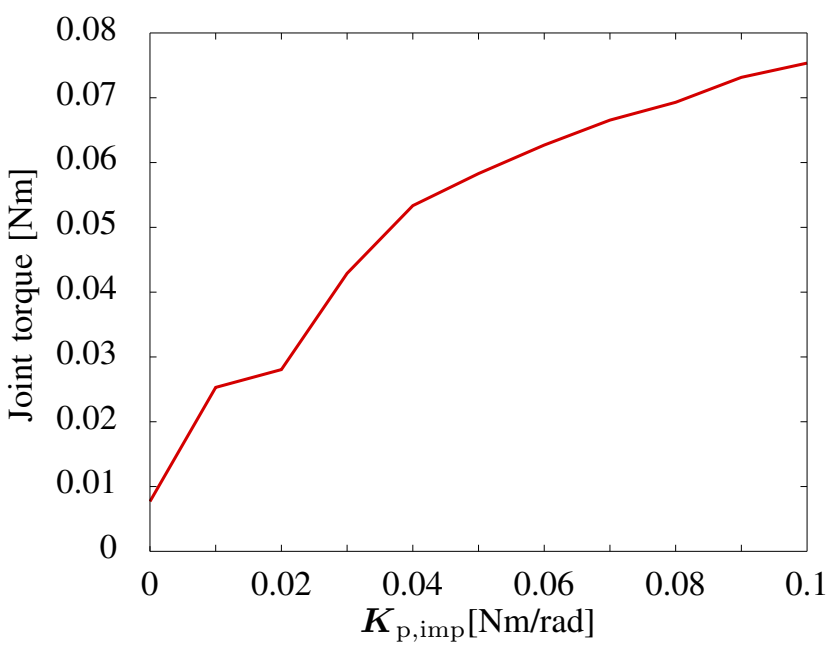

Fig. 9. Experiments: verification of the impedance behavior. The curve depicts the joint torque generated by the tendon forces depending on the desired impedance stiffness. A position error of the joint is imposed externally by a mechanical fixture.

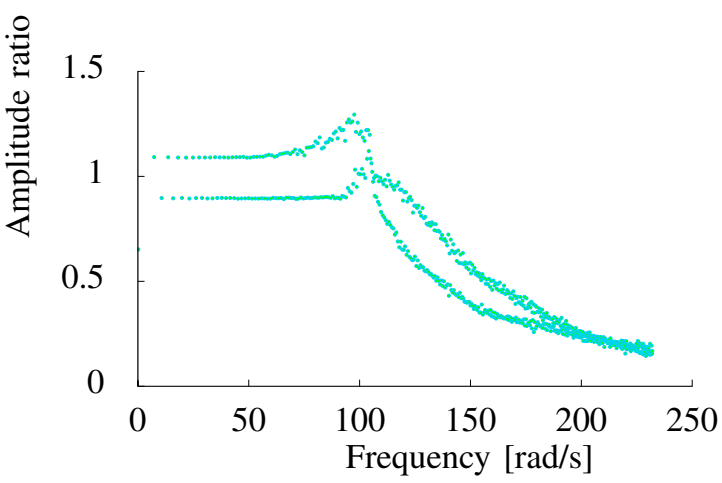

Fig. 10. Experiments: Gain diagram for the PIP joint controlled by the backstepping controller (indicated by light blue dots). The pretension was set to $20 \mathrm{~N}$.

of the controller was established mathematically, a set of experiments and simulations were conducted to verify the applicability of the controller. The experiments demonstrate the excellent performance of the controller. It is interesting to note that, because of the torque sharing approach, the system is able to perform with only one motor. The on going work concentrates on extending the controller to include the stiffness adjustment dynamics, that is to design a multiple input multiple output (MIMO) backstepping controller.

\section{REFERENCES}

[1] D. J. Bennett, "What are the advantages of variable stiffness control?" IEEE, Engineering in Medicine and Biology Magazine, vol. 11, no. 4, pp. 86-87, Dec. 1992.

[2] A. Albu-Schäffer, O. Eiberger, M. Grebenstein, S. Haddadin, C. Ott, T. Wimböck, S. Wolf, and G. Hirzinger, "Soft robotics: From torque 
feedback controlled lightweight robots to intrinsically compliant systems," IEEE Robotics and Automation Magazine, vol. 15, no. 3, pp. 20-30, 2008

[3] C. Ott, A. Albu-Schäffer, A. Kugi, and G. Hirzinger, Cutting Edge Robotics. Advanced Robotic Systems International, pro literatur Verlag, 2005, ch. Cartesian Impedance Control of Flexible Joint Robots: A Decoupling Approach, pp. 671-682.

[4] - "Decoupling based cartesian impedance control of flexible joint robots," Robotics and Automation, IEEE International Conference on, pp. 3101-3107, 2003.

[5] S. Nicosia and P. Tomei, "A method to design adaptive controller for flexible joint robots," Robotics and Automation, IEEE International Conference on, pp. 701-706, 1992.

[6] A. Albu-Schäffer, Ch. Ott, and G. Hirzinger, "A unified passivity based control framework for position, torque and impedance control of flexible joint robots," International Journal of Robotics Research, vol. 26, no. 1, pp. 23 - 39, January 2007.

[7] P. V. Kokotović, H. K. Khalil, and J. O'Reilly, Singular Perturbation Methods in Control: Analysis and Design. Academic Press Inc., 1986.

[8] E. P. Antonio Loria, "Cascaded nonlinear time-varying systems: analysis and design," Advanced topics in control systems theory. Conference, Paris , FRANCE, Tech. Rep., 2004.

[9] P. Kokotovic and M. Arcak, "Constructive nonlinear control: A historical perspective," Automatica, vol. 37, pp. 637-662, 2001.

[10] A. De Luca, R. Farina, and P. Lucibello, "On the control of robots with visco-elastic joints," Robotics and Automation, IEEE International Conference on, vol. 1, pp. 4297-4302, 2005.

[11] V. Potkonjak, B. Svetozarevic, K. Jovanovic, and O. Holland, "The Puller-Follower Control of Compliant and Noncompliant Antagonistic Tendon Drives in Robotic Systems," Advanced Robotic Systems, International Journal of, vol. 8, no. 5, pp. 143-155, 2012.

[12] V. Utkin, "Sliding mode control design principles and applications to electric drives," Industrial Electronics, IEEE Transactions on, vol. 40, no. 1 , pp. $23-36$, feb 1993.

[13] S.-H. Huh and Z. Bien, "Robust sliding mode control of a robot manipulator based on variable structure-model reference adaptive control approach," IET Control Theory Applications, vol. 1, no. 5, pp. 13551363, Sep. 2007.

[14] B. Song and J. K. Hedrick, Dynamic Surface Control of Uncertain Nonlinear Systems, An LMI Approach. Springer London, 2013.

[15] H. Kobayashi and R. Ozawa, "Adaptive neural network control of tendon-driven mechanisms with elastic tendons," Automatica, vol. 39, pp. 1509-1519, 2003.

[16] S. Huh, G. Tonietti, and A. Bicchi, "Neural network based robust adaptive control for a variable stiffness actuator," Control and Automation, Mediterranean Conference on, pp. 1028-1034, Jun. 2008.

[17] R. Platt, M. Abdallah, C. Wampler, and B. Hargrave, "Joint-space torque and stiffness control of tendon-driven manipulators," Intelligent Robots and Systems, IEEE International Conference on, vol. 1, pp. 74-79, 2010.

[18] M. Abdallah, , U. Warren, MI, R. Platt, B. Hargrave, and F. Permenter, "Position control of tendon-driven fingers," Robotics and Automation, IEEE International Conference on, vol. 1, pp. 2859 - 2864, 2012.

[19] R. Schiavi, G. Grioli, S. Sen, and A. Bicchi, "VSA-II: a novel prototype of variable stiffness actuator for safe and performing robots interacting with humans," Robotics and Automation, IEEE International Conference on, pp. 2171-2176, May 19-23, 2008.

[20] T. Wimboeck, C. Ott, A. Albu-Schaeffer, and G. Hirzinger, "Impedance control for variable stiffness mechanisms with nonlinear joint coupling," Intelligent Robots and Systems, IEEE International Conference on, vol. 1, pp. 3796-3803, 2008.

[21] G. Palli, "Model and control of tendon actuated robots," Ph.D. dissertation, DEIS, University of Bologna, 2007.

[22] G. Palli and C. Melchiorri, "Robust control of robots with variable joint stiffness," Advanced Robotic, International Conference on, pp. 1-6, Jun. 22-26, 2009.

[23] C. Ott, Cartesian Impedance Control of Redundant and Flexible-Joint Robots. Springer Tracts in Advanced Robotics (STAR), 2008, vol. 49.

[24] M. Spong, New Trends and Applications of Distributed Parameter Systems. Marcel Dekker, New York, 1990, ch. The Control of Flexible Joint Robots: A Survey, pp. 355-383.

[25] B. Paden and R. Panja, "Globally asymptotically stable 'pd+' controller for robot manipulators," International Journal of Control, vol. 47, no. 6, pp. 1697-1712, 1988.

[26] M. Chalon, W. Frield, J. Reinecke, T. Wimböck, and A. Albu-Schäffer, "Impedance control of a non-linearly coupled tendon driven thumb," in Intelligent Robots and Systems, IEEE International Conference on, 2011, pp. 4215-4221.

[27] H. Kobayashi, K. Hyodo, and D. Ogane, "On tendon-driven robotic mechanisms with redundant tendons," International Journal of Robotic Research, vol. 17, no. 4, pp. 561-571, 1998.

[28] M. Chalon, T. Wimböck, and G. Hirzinger, "Torque and stiffness workspace analysis for flexible tendon mechanisms," in Robotics and Automation, IEEE International Conference on, 2010.

[29] W. Friedl, J. Reinecke, M. Chalon, and G. Hirzinger, "FAS A Flexible Antagonistic Spring element for a high performance tendon driven hand," Intelligent Robots and Systems, IEEE International Conference on, vol. 1, pp. 1366 - 1372, 2011.

[30] M. Grebenstein and P. van der Smagt, "Antagonism for a highly anthropomorphic hand arm system," Advanced Robotics, vol. 22, no. 22 , pp. 39-55, 2008.

[31] S. Donghoon, K. Wonhee, and C. C. Choo, "Position control of a permanent magnet stepper motor by miso backstepping in semistrict feedback form," Advanced Intelligent Mechatronics, IEEE/ASME International Conference on, pp. 808-813, 2011.

[32] R. Balasubramanian and Y. Matsuoka, "Biological stiffness control strategies for the Anatomically Correct Testbed (ACT) hand," in Robotics and Automation, IEEE International Conference on, May 1923, 2008, pp. 737-742.

[33] M. Grebenstein, A. Albu-Schäffer, T. Bahls, M. Chalon, O. Eiberger, W. Friedl, R. Gruber, U. Hagn, R. Haslinger, H. Höppner, S. Jörg, M. Nickl, A. Nothhelfer, F. Petit, J. Reill, N. Seitz, T. Wimbock, S. Wolf, T. Wüsthoff, and G. Hirzinger, "The dlr hand arm system," Robotics and Automation, IEEE International Conference on, 2011. 